\title{
Micropapillary pattern and grade of stromal invasion in pT1 adenocarcinoma of the lung: usefulness as prognostic factors
}

\author{
Takehito Kawakami ${ }^{1,2}$, Kazuki Nabeshima ${ }^{1}$, Yoshifumi Makimoto ${ }^{1,2}$, Makoto Hamasaki ${ }^{1}$, \\ Akinori Iwasaki ${ }^{2}$, Takayuki Shirakusa ${ }^{2}$ and Hiroshi Iwasaki ${ }^{1}$ \\ ${ }^{1}$ Department of Pathology, Fukuoka University School of Medicine, Fukuoka, Japan and ${ }^{2}$ Second Department \\ of Surgery, Fukuoka University School of Medicine, Fukuoka, Japan
}

\begin{abstract}
Recently, the stromal invasion grading system was proposed for small adenocarcinomas of $\leq 2.0 \mathrm{~cm}$. The system is based on the presence or absence of a fibrotic focus, and the extent of invasion into the fibrotic focus. Although stromal invasion grading system closely correlated with the prognosis, stromal invasion grade 3 , representing stromal invasion into the center of a fibrotic focus, was the largest group of tumors and showed variable prognosis. In this study, we investigated whether stromal invasion grading system could be applied to and validated in pT1 adenocarcinomas as the TNM classification is the most universally used system. Furthermore, we investigated whether stromal invasion grade 3 cases could be subclassified according to the presence and absence of micropapillary pattern. The study included 120 cases of pT1 lung adenocarcinomas, of which $81(68 \%)$ cases were stromal invasion grade 3 . Micropapillary pattern was positive in $80 \%$ of grade 3 cases. For stromal invasion grade 3 cases, the 5-year survival rate of patients with micropapillary patternpositive carcinomas was $63 \%$, which was significantly worse than $94 \%$ of those with micropapillary patternnegative carcinomas $(P=0.0196)$. The latter was very close to that for patients with stromal invasion grade $0-2$ $(95 \%)$. Moreover, small cluster invasion was observed at sites of stromal invasion significantly more often in micropapillary pattern-positive cases than negative cases. Thus, the stromal invasion grading system is reproducible and correlates with prognosis even in pT1 lung adenocarcinomas. Moreover, among patients with stromal invasion grade 3 carcinomas, favorable prognosis is noted in micropapillary pattern-negative cases. The micropapillary pattern subclassification provides an advantage to the stromal invasion grading system and reconfirms the importance of micropapillary pattern as a prognostic marker. Our study is the first to point to the possible association of micropapillary pattern-positive carcinomas and small cluster invasion.

Modern Pathology (2007) 20, 514-521. doi:10.1038/modpathol.3800765; published online 2 March 2007
\end{abstract}

Keywords: lung adenocarcinoma; pT1; micropapillary pattern; stromal invasion; prognosis

Lung cancer is one of the most common causes of cancer-related deaths in the United States and other industrialized countries, including Japan. ${ }^{1,2}$ Among the major histopathological types of the lung carcinoma, the incidence of adenocarcinoma has been increasing in recent years. ${ }^{3}$ Owing to recent technological improvements in high-resolution computed tomography and the introduction of computed tomography screening, the incidence of small-sized pathological-T1 (pT1) cases is on the rise among surgically resected adenocarcinoma

Correspondence: Dr T Kawakami, MD, Department of Pathology, Fukuoka University School of Medicine, 7-45-1 Nanakuma, Jonan-ku, Fukuoka 814-0180, Japan.

E-mail: goujinn@yahoo.co.jp

Received 04 December 2006; revised and accepted 22 January 2007; published online 2 March 2007 cases., ${ }^{4,5}$ Although bronchioloalveolar carcinoma is reported as the only subtype without any invasive features and shows an excellent prognosis, some of the other pT1 adenocarcinoma cases may develop distant metastasis in a short period after complete surgical resection. ${ }^{6-8}$ Thus, although the tumornode-metastasis (TNM) stage and the pathological stage, including the presence or absence of lymph node metastasis, have been the most reliable prognostic factors in patients with lung carcinoma, ${ }^{9,10}$ it is crucial to identify additional prognostic factors within each given stage to select most appropriate therapy and individualize the follow-up frequency.

Recently, Sakurai et $a l^{11}$ have histopathologically graded the degree of invasion in small adenocarcinomas of $\leq 2.0 \mathrm{~cm}$ and explored the relationship between invasion and prognosis of patients with adenocarcinomas. Although their stromal invasion 
grading system closely correlated with the prognosis, their stromal invasion grade 3 , representing stromal invasion into the center of a fibrotic focus, was the largest group of tumors (212/380 cases, $56 \%$ ) and showed variable prognosis. Therefore, it would be favorable to subclassify this most heterogeneous group. In contrast, lung adenocarcinomas with a micropapillary pattern have been reported to have a worse biological behavior, and that the patients with micropapillary pattern tend to present with advanced stage including lymph node metastasis. $^{12-15}$

In the present study, we investigated whether the stromal invasion grading system correlates with prognosis including pT1 $(\leq 3.0 \mathrm{~cm})$ adenocarcinomas. Furthermore, we also tested whether the stromal invasion grade 3 could be subclassified into two groups that show significantly different prognoses based on the presence and absence of micropapillary pattern. Finally, we examined whether micropapillary pattern was frequently associated with any mode of stromal invasion.

\section{Materials and methods}

\section{Patients}

We reviewed 134 cases of pT1 lung adenocarcinoma that had been surgically resected at the Second Department of Surgery, Fukuoka University Hospital between April 1993 and December 2002. Anonymous use of redundant tissue is part of the standard treatment agreement with patients in our hospital when no objection was expressed. The pathological stage was determined according to the TNM classification of malignant tumors (UICC). ${ }^{10}$ pT1 tumors were defined as 'tumors of $3 \mathrm{~cm}$ or less in the greatest dimension, being surrounded by lung tissue or visceral pleura, and not involving the main bronchus'. ${ }^{10}$ Of the 134 cases, seven cases lost in the follow-up were excluded from the survival analysis. In addition, seven cases of invasive adenocarcinoma with no central fibrotic focus (mostly so-called 'solid adenocarcinoma') were excluded from stromal invasion grade analysis. The remaining 120 cases were studied to explore the relationship between stromal invasion, micropapillary patterns and prognosis. All patients underwent complete resection of the tumors. The mean follow-up period was 75 months (range 2-117 months).

\section{Pathologic Evaluation}

The surgically resected specimens were fixed routinely in $10 \%$ formalin, and the whole tumor nodules were processed into paraffin blocks for histopathological examination. Tissue sections were cut $4 \mu \mathrm{m}$ thick, including the largest cut surface of the tumor, and stained with hematoxylin and eosin (H \& E) and elastica-van-Gieson stain (EvG). For MUC1 immu- nohistochemistry, $4 \mu \mathrm{m}$ sections were mounted on MAS (Matsunami Adhesive Silane)-coated glass slides, deparaffinized, and autoclaved for $10 \mathrm{~min}$ at $121^{\circ} \mathrm{C}$ in the presence of $10 \mathrm{mM}$ Sodium-citrate buffer ( $\mathrm{pH}$ 6.0) solution to improve the immunoreactivity of the antigen. A mouse monoclonal antibody directed against MUC1 (Novocastra Laboratories, Newcastle, UK) was used as the primary antibody at a 1:100 dilution. Immunohistochemical staining was performed by the LSAB (labeled streptavidin-biotin-peroxidase) method (Dako, Glostrup, Denmark). Alkaline phosphatase activity was visualized by naphthol-AS-BI-phosphate as a substrate (Sigma, St Louis, MO, USA) and new fuchsin as a coupler (Merck, Darmstadt, Germany).

The histopathological type was determined according to the WHO classifications $2004,{ }^{16}$ and stromal invasion grade was evaluated as described previously. ${ }^{11}$ Briefly, stromal invasion grade 0 was defined as pure bronchioloalveolar carcinoma, which shows no evidence of stromal invasion (Figure 1a); stromal invasion grade 1 as stromal invasion in the area of bronchioloalveolar carcinoma growth (Figure 1b); stromal invasion grade 2 as stromal invasion localized on the periphery of a fibrotic focus (Figure 1c); and stromal invasion grade 3 as stromal invasion into the center of a fibrotic focus (Figure 1d). Stromal invasion was confirmed by EvG staining, which demonstrated structural deformity of the stromal elastic fiber framework at the sites of invasion. ${ }^{11,17}$

Lung adenocarcinomas show two types of papillary structures: papillary structure and micropapillary pattern. The papillary structure is characterized by a complex papillary glandular proliferation along fibrovascular cores, ${ }^{16}$ whereas micropapillary pattern is defined as papillary structures with tufts lacking a central fibrovascular core (Figure 2a). ${ }^{12}$ The outer surface of the micropapillary cell clusters showed expression of cell surface glycoprotein MUC1 (Figure 2b), and in the ordinary portions of lung adenocarcinoma MUC1 labeling was limited to the luminal surface of carcinoma cells (Figure 2c). The extent of micropapillary pattern was determined as none ( $0 \%$ of the tumor), $1+(<10 \%), 2+$ $(10-50 \%)$, or $3+(>50 \%)$ based on the areas of micropapillary pattern in tumors. Furthermore, cases of none and $1+$ were classified as micropapillary pattern-negative, whereas those of $2+$ and $3+$ as micropapillary pattern-positive as described previously. ${ }^{15}$ This micropapillary pattern scoring was performed independently by two pathologists (TK and $\mathrm{KN}$ ), and a high level of concordance $(>90 \%)$ was achieved. In case of disagreement, the slides were reviewed again to obtain a consensus view.

The clinicopathological parameters considered in this study included age, gender, tumor size, lymph node metastasis, pleural invasion and small cluster invasion. The latter was defined as markedly 

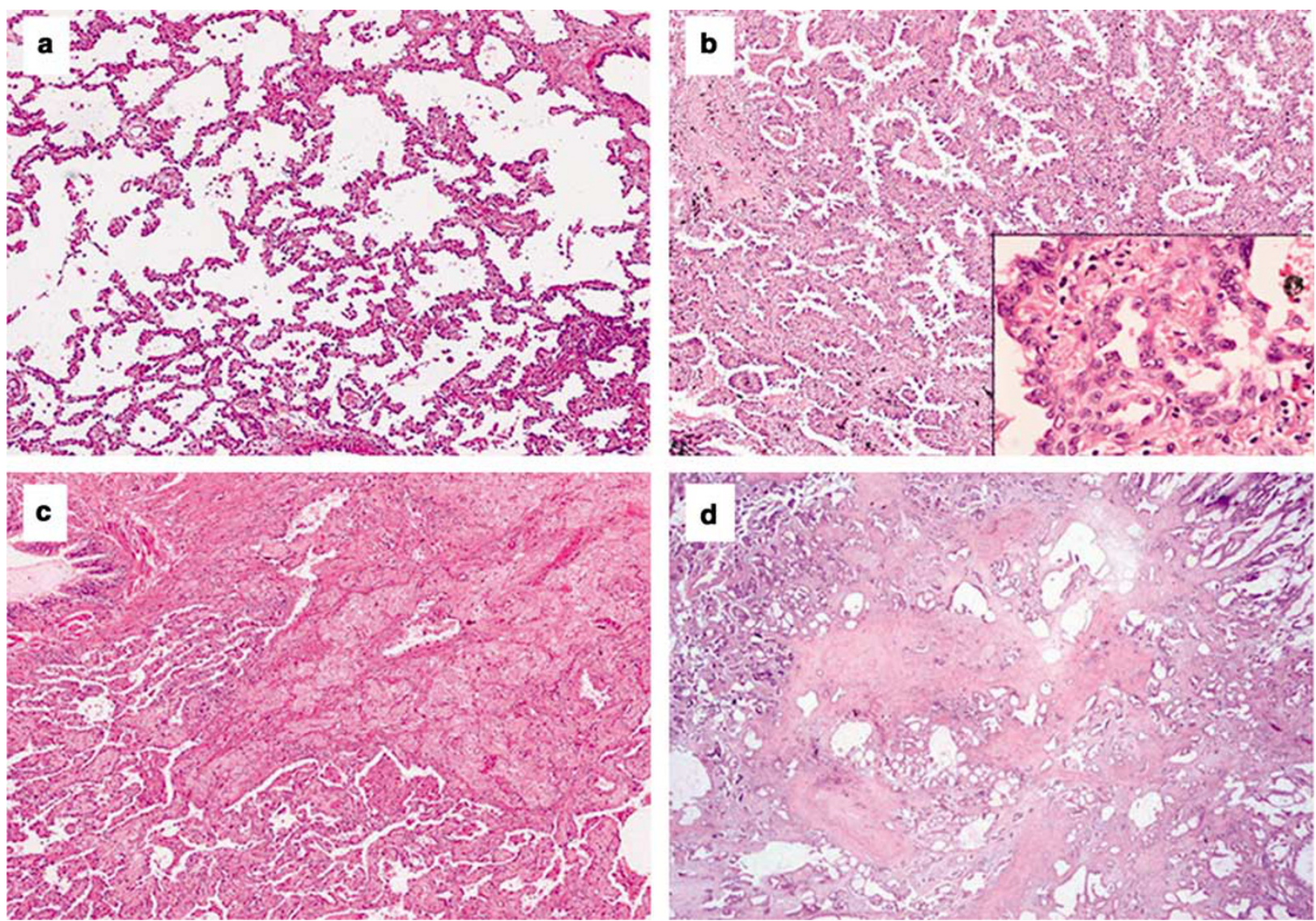

Figure 1 Microphotographs of lung adenocarcinomas of different grades. (a) Grade 0 invasion. The carcinoma shows a bronchioloalveolar growth pattern with no stromal invasion. (b) Grade 1 invasion. The carcinoma shows stromal invasion in the area of bronchioloalveolar growth. An inset shows areas of stromal invasion at higher power. (c) Grade 2 invasion. The carcinoma shows stromal invasion localized on the periphery of a central fibrosis. (d) Grade 3 invasion. The carcinoma shows stromal invasion extending into the center of a fibrotic focus. (a-d) HE, original magnification $\times 40$. (b, inset) HE, original magnification $\times 400$.

resolved acinar or papillary structures of tumors with small clusters of carcinoma cells lying ahead and invading the stroma within the fibrotic focus (Figure 2d), which is very similar to tumor budding in colorectal carcinomas. ${ }^{18}$

\section{Statistical Analysis}

Summary statistics were obtained using established methods and statistical analysis software StatView for Windows version 5.0 (SAS Institute Inc., Cary, NC, USA). The relationships between several clinicopathological parameters and histopathological subgroups were evaluated using the $\chi^{2}$ test and Fisher's exact test. Survival curves were plotted using the Kaplan-Meier method, and $P$-values were calculated using the log-rank test. Multivariate analysis was performed by Cox regression. A $P$-value of $<0.05$ was considered statistically significant.

\section{Results}

\section{Clinical Findings}

The clinicopathological characteristics of the 120 patients (49 males, 71 females; age range, 28-85 (mean $=64.8)$ years) are summarized in Table 1. Lobectomy was performed in 88 patients $(73 \%)$. The remaining 32 patients underwent limited surgery (segmentectomy or partial resection). Pathologically, 101 patients were classified as p-Stage IA, six patients as p-Stage IIA, and 13 as p-Stage IIIA. The majority of stromal invasion grade $0-2$ and stromal invasion grade 3 without micropapillary pattern belonged to p-Stage IA (92 and 100\%, respectively), whereas about $25 \%$ of stromal invasion grade 3 with micropapillary pattern were classified as p-Stage IIA or IIIA, indicating that more cases with lymph node metastasis were included in the stromal invasion grade 3 with micropapillary pattern group.

\section{Histopathological Findings}

Eight cases were classified as bronchioloalveolar carcinoma $(7 \%)$, two as acinar $(2 \%)$, five as papillary $(4 \%)$, and the remaining 105 patients as mixed subtypes (88\%). The relationship between stromal invasion grade and micropapillary pattern is summarized in Table 2. Grades 0, 1 and 2 stromal 

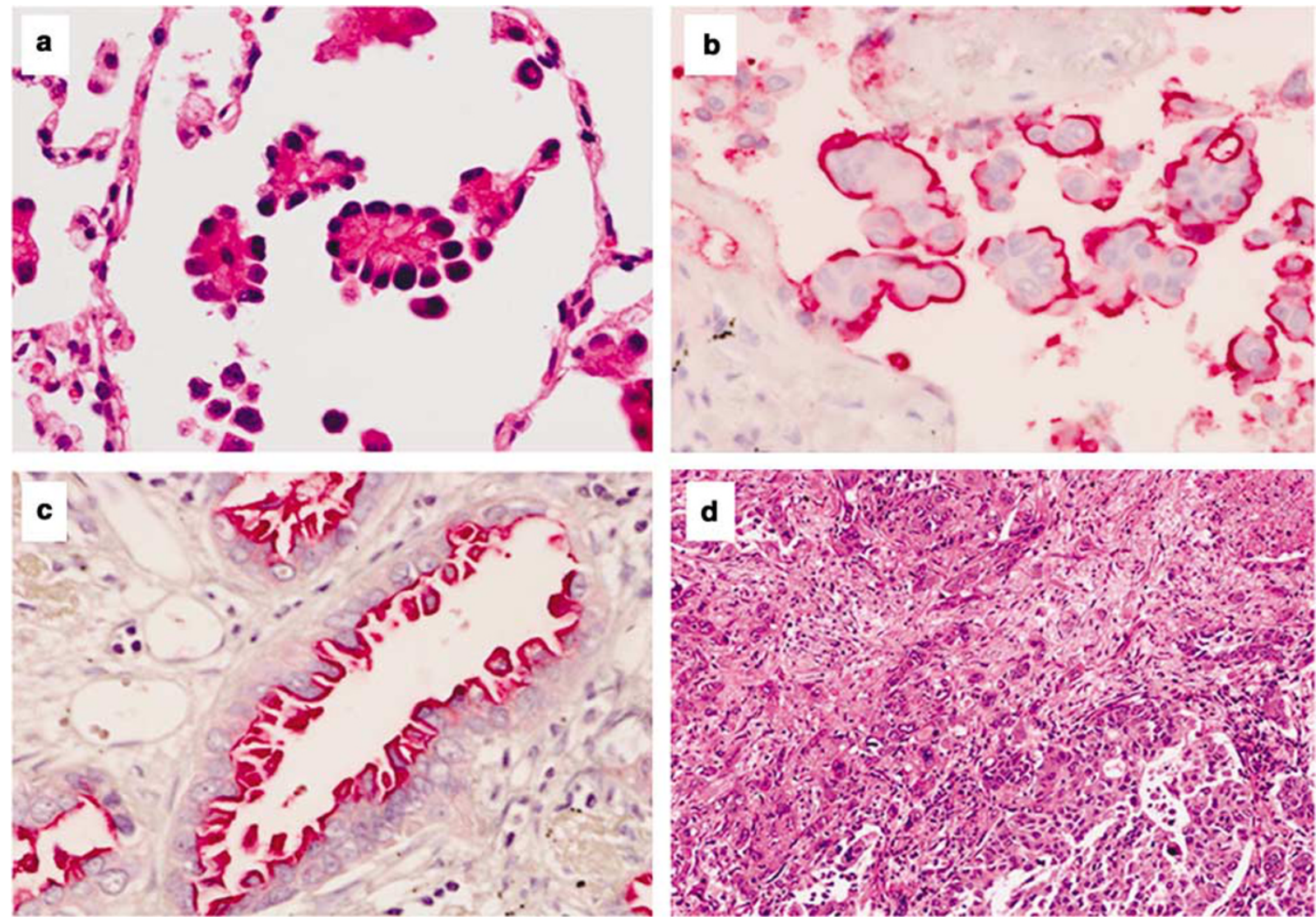

Figure 2 Histopathological features of micropapillary pattern. (a) Micropapillary tufts lacking a central fibrovascular core. (b) Immunohistochemical demonstration of MUC 1 on the outer surface of micropapillary tufts. (c) Ordinary (non-micropapillary) portions of the lung adenocarcinoma showing MUC1 reactivity limited to the luminal surface of carcinoma cells. (d) Small cluster invasion, observed as isolated small clusters of invading carcinoma cells in the fibrotic focus. (a, d) HE, original magnification (a) $\times 400$, (d) $\times 100$; (b, c) MUC 1, original magnification $\times 400$.

invasions were observed in $8(7 \%), 21(18 \%)$ and 10 $(8 \%)$ cases, respectively, whereas grade 3 was noted in the majority of the cases $(81 / 120$ cases, $68 \%)$. As the stromal invasion grade increased, more micropapillary pattern cases were found: no micropapillary pattern-positive $(2+$ and $3+)$ cases among grade 0 and only $38 \%$ (8/21 cases) among grade 1 , compared with $70 \%$ (7/10 cases) and $80 \%$ (65/81 cases) of grade 2 and 3, respectively.

Table 3 shows the relationship between micropapillary pattern and clinicopathological characteristics. No micropapillary pattern was found in $21 / 120(18 \%)$, and $1+, 2+$ and $3+$ of micropapillary pattern were noted in 19/120 (16\%), 54/120 $(45 \%)$ and $26 / 120(22 \%)$, respectively. The overall micropapillary pattern-positive $(2+$ and $3+)$ cases were $67 \%$ (80/120 cases). In the micropapillary pattern-positive cases, tumor size was more frequently larger than $1 \mathrm{~cm}$ in diameter, and lymph node metastasis, pleural invasion and small cluster invasion at the sites of stromal invasion were more frequently observed than in micropapillary patternnegative cases; the differences were statistically significant. Age and gender were similar in the two groups.

\section{Correlation of SIG and MPP with Clinical Outcome}

Survival rates

The overall 5-year survival rates for patients with stromal invasion grade $0-2$ and stromal invasion grade 3 carcinomas were 95 and 69\%, respectively, and the difference was statistically significant $(P=0.0048$, Figure 3$)$. These results were similar to those reported for tumors measuring $\leq 2.0 \mathrm{~cm}$ by Sakurai et al. ${ }^{11}$ Thus, stromal invasion grade 3 is also the important poor prognostic factor in lung pT1 adenocarcinomas. However, stromal invasion grade 3 comprised the majority of the pT1 adenocarcinoma cases $(81 / 120$ cases, $68 \%)$. As we speculated that this stromal invasion grade group could include patients with variable prognoses, we investigated whether micropapillary pattern could be an additional histological marker for subclassification of the stromal invasion grade 3 group. Figure 4 shows the overall survival curves of patients with stromal invasion grade 3 divided into four groups based on extents of micropapillary pattern. The 5year survival rate of patients with micropapillary pattern-positive $(2+$ and $3+)$ carcinomas $(65 / 81$ cases, $80 \%$ ) was $63 \%$, which was significantly 
Table 1 Characteristics of patients with pT1 adenocarcmoma

\begin{tabular}{|c|c|c|c|c|c|}
\hline \multirow[t]{2}{*}{ Characteristics } & \multirow[t]{2}{*}{ All cases $(\%)$} & \multicolumn{2}{|c|}{ Stromal invasion grade $0-2$ cases } & \multicolumn{2}{|c|}{ Stromal invasion grade 3 cases } \\
\hline & & \multicolumn{2}{|c|}{ Micropapillary pattern (\%) } & \multicolumn{2}{|c|}{ Micropapillary pattern (\%) } \\
\hline No. of patients & 120 & 24 & 15 & 16 & 65 \\
\hline \multicolumn{6}{|l|}{ Age (year) } \\
\hline Mean & 64.8 & 61.8 & 63.1 & 68.9 & 64.8 \\
\hline Range & $28-85$ & $29-78$ & $51-85$ & $28-85$ & $29-85$ \\
\hline \multicolumn{6}{|l|}{ Gender } \\
\hline Male & $49(41)$ & $7(29)$ & $8(53)$ & $6(38)$ & $28(43)$ \\
\hline Female & $71(59)$ & $17(71)$ & $7(47)$ & $10(63)$ & $37(57)$ \\
\hline \multicolumn{6}{|l|}{ Operative mode } \\
\hline Lobectomy & $86(72)$ & $12(50)$ & $12(80)$ & $13(81)$ & $49(75)$ \\
\hline Limited surgery & $34(28)$ & $12(50)$ & $3(20)$ & $3(19)$ & $16(25)$ \\
\hline \multicolumn{6}{|l|}{ Histologic subtypes } \\
\hline Bronchioloalveolar & $8(7)$ & $8(33)$ & $0(0)$ & $0(0)$ & $0(0)$ \\
\hline Acinar & $2(2)$ & $0(0)$ & $0(0)$ & $2(13)$ & $0(0)$ \\
\hline Papillary & $5(4)$ & $3(13)$ & $2(13)$ & $0(0)$ & $0(0)$ \\
\hline Mixed subtypes & $105(88)$ & $13(54)$ & $13(87)$ & $14(88)$ & $65(100)$ \\
\hline \multicolumn{6}{|l|}{ Pathological stage } \\
\hline IA & $101(84)$ & $23(96)$ & $13(87)$ & $16(100)$ & $49(75)$ \\
\hline IIA & $6(5)$ & $1(4)$ & $1(7)$ & $0(0)$ & $4(6)$ \\
\hline IIIA & $13(11)$ & $0(0)$ & $1(7)$ & $0(0)$ & $12(18)$ \\
\hline
\end{tabular}

Table 2 Relationship between grade of stromal invasion and micropapillary pattern

\begin{tabular}{|c|c|c|c|c|c|}
\hline \multirow[t]{2}{*}{ Stromal invasion grade } & \multirow[t]{2}{*}{ No. of cases } & \multicolumn{2}{|c|}{ Micropapillary pattern-negative group (\%) } & \multicolumn{2}{|c|}{ Micropapillary pattern—positive group (\%) } \\
\hline & & 0 & $1+$ & $2+$ & $3+$ \\
\hline Total & 120 & 21 (18) & 19 (16) & $54(45)$ & $26(22)$ \\
\hline Grade 0 & 8 & 8 (100) & $0(0)$ & $0(0)$ & $0(0)$ \\
\hline Grade 1 & 21 & 7 (33) & $6(29)$ & 7 (33) & $1(5)$ \\
\hline Grade 2 & 10 & $1(10)$ & $2(20)$ & $4(40)$ & 3 (30) \\
\hline Grade 3 & 81 & $5(6)$ & $11(14)$ & $43(53)$ & $22(27)$ \\
\hline
\end{tabular}

worse than $94 \%$ of those with micropapillary pattern-negative (None and $1+$ ) carcinomas (16/ $81,20 \%, P=0.0196)$. The 5-year survival rate for micropapillary pattern-negative stromal invasion grade 3 patients was very close to that for patients with stromal invasion grade $0-2(95 \%)$. As correlation of the micropapillary pattern with worse prognosis was suggested, univariate and multivariate analyses were performed including stratification for stage.

Univariate analyses

Carcinoma cases included in this study belonged to p-Stage IA, IIA or IIIA (Table 1). Within clinicopathological parameters of p-Stage IA carcinomas, univariate analysis identified the significant association of micropapillary pattern (positive) and stromal invasion grade (grade 3) with the poor patient survival (Table 4, micropapillary pattern,
$P=0.0317$; stromal invasion grade, $P=0.0332$ ). No significant parameters were identified in p-Stage IIA and IIIA carcinomas.

Multivariate analyses

Both factors identified as significant by univariate analyses were entered into Cox multivariate regression analysis. This analysis identified micropapillary pattern (positive) as a significant predictor and independent factor for a shorter overall survival in p-Stage IA carcinoma cases (Table 5, micropapillary pattern, $P=0.0493)$.

\section{Discussion}

Our study showed that the stromal invasion grading system proposed by Sakurai et $a l^{11}$ is reproducible and valid even in pT1 lung adenocarcinomas. 
Table 3 Micropapillary pattern and clinicopathological characteristics

\begin{tabular}{|c|c|c|c|}
\hline & $\begin{array}{l}\text { Micropapillary } \\
\text { pattern- } \\
\text { positive }(\%)\end{array}$ & $\begin{array}{l}\text { Micropapillary } \\
\text { pattern- } \\
\text { negative }(\%)\end{array}$ & $\mathrm{P}$-value \\
\hline \multicolumn{4}{|l|}{ Age $(n=120)$} \\
\hline$<70(n=81)$ & $56(69)$ & $25(31)$ & \\
\hline$\geq 70(n=39)$ & $24(62)$ & $15(38)$ & 0.4083 \\
\hline \multicolumn{4}{|l|}{ Gender $(n=120)$} \\
\hline Male $(n=49)$ & $36(73)$ & $13(27)$ & \\
\hline Female $(n=71)$ & $44(62)$ & $27(38)$ & 0.1891 \\
\hline \multicolumn{4}{|l|}{ Tumor size $(n=120)$} \\
\hline$\leq 1 \mathrm{~cm}(n=14)$ & $6(43)$ & $8(5)$ & \\
\hline$>1 \mathrm{~cm}(n=106)$ & $74(70)$ & $32(30)$ & 0.0444 \\
\hline \multicolumn{4}{|c|}{ Lymph node metastasis $(n=120)$} \\
\hline Positive $(n=19)$ & $18(95)$ & $1(5)$ & \\
\hline Negative $(n=101)$ & $62(61)$ & $39(39)$ & 0.0047 \\
\hline \multicolumn{4}{|c|}{ Pleural invasion $(n=120)$} \\
\hline Positive $(n=49)$ & $38(78)$ & $11(22)$ & \\
\hline Negative $(n=71)$ & $42(59)$ & $29(41)$ & 0.0356 \\
\hline \multicolumn{4}{|c|}{ Small cluster invasion $(n=120)$} \\
\hline Positive $(n=70)$ & $63(90)$ & $7(10)$ & \\
\hline Negative $(n=50)$ & $17(34)$ & $33(66)$ & 0.0051 \\
\hline
\end{tabular}

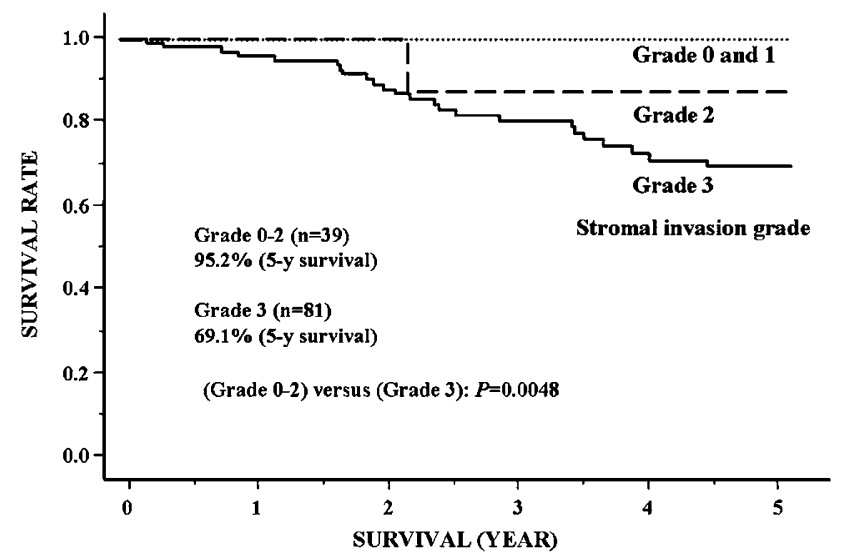

Figure 3 Survival curves according to histopathological grade of stromal invasion (grades $0-3$ ). The 5-year survival rate of patients with stromal invasion grade $0-2$ carcinomas was significantly higher than that of patients with stromal invasion grade 3 carcinomas (log rank test, $P=0.0048$ ).

Sakurai et al ${ }^{11}$ studied small adenocarcinomas of $\leq 2.0 \mathrm{~cm}$ to establish the concept of 'curable' lung cancer and showed that stromal invasion grade 0 and 2 could be considered 'minimally invasive' or 'early' adenocarcinomas. We investigated whether their stromal invasion grading system could be applied to pT1 adenocarcinomas because the TNM classification is most universally used and pT1 tumors are the smallest tumors in the classification.

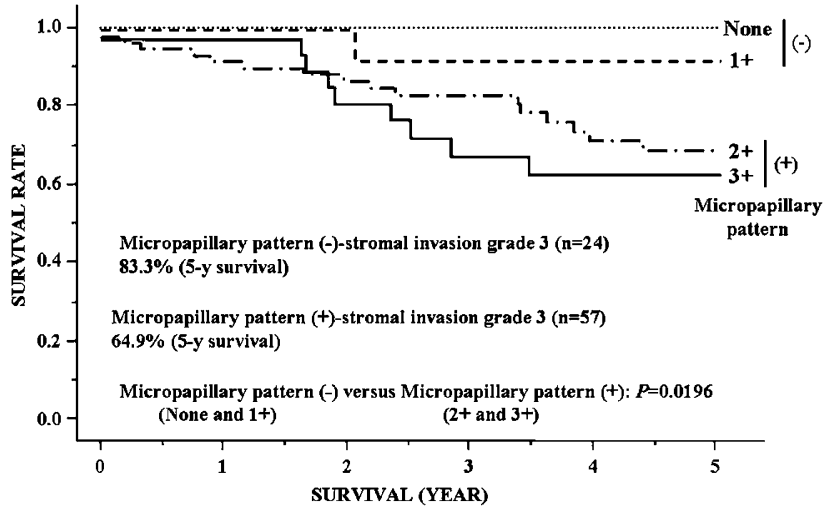

Figure 4 Survival rates of patients with stromal invasion grade 3 carcinomas according to presence or absence of a micropapillary pattern. The prognosis of patients with micropapillary patternnegative (None and $1+$ ) stromal invasion grade 3 adenocarcinoma was significantly better than those with micropapillary pattern-positive $(2+$ and $3+)$ tumors (log rank test, $P=0.0196)$.

In addition, our results solved a problem in their grading system. Stromal invasion grade 3 , which comprised the largest group in their classification and included patients with variable prognoses, was subclassified in our study into two groups based on the presence and absence of micropapillary pattern. Micropapillary pattern-negative stromal invasion grade 3 cases showed a much better 5 -year survival rate compared with micropapillary pattern-positive stromal invasion grade 3 cases (94 vs $63 \%$ ). This indicates that when micropapillary pattern is negative even patients of the stromal invasion grade 3 group with full stromal invasion could have better prognosis, which was similar to that of stromal invasion grade 0-2 groups with none or limited stromal invasion. This subclassification has provided an advantage for the use of stromal invasion grading system of Sakurai et al, and at the same time highlighted the importance of micropapillary pattern in assessing the progression of lung adenocarcinoma.

The prognostic significance of central fibrosis or scar of adenocarcinoma of the lung has been reported by several investigators. ${ }^{7,11,17,19-22}$ Shimosato et $a 1^{19}$ proposed that the central scar in carcinomas was a secondary phenomenon rather than a precursor of carcinoma, and demonstrated that the degree of collagenization in the fibrotic focus closely correlated with tumor progression and prognosis. Others indicated that the $\operatorname{size}^{21}$ and characteristics of the central fibrosis, such as the interface pattern,${ }^{20}$ the presence of active fibroblast proliferation, ${ }^{7}$ the pattern of stromal elastic frameworks, ${ }^{17}$ and the extent of desmoplasia ${ }^{22}$ are significant prognostic factors. The stromal invasion grading system is also based on the presence and absence of a fibrotic focus and the extent of invasion into the fibrotic focus. ${ }^{11}$ Each grade was clearly defined, so that we could demonstrate the 
Table 4 correlation fo micropapillary pattern, stromal invasion grade, small cluster invasion and pleural invasion with overall survival by univariate analysis in p-Stage IA cases

Variable

Correlation with survial $\left(\mathrm{P}^{\mathrm{a}}\right)$

Micropapillary pattern (positive)

Stromal invasion grade (grade 3)

Small cluster invasion (positive)

Pleural invasion (positive)

0.0317

0.0332

0.0731

0.2003

${ }^{\mathrm{a}}$ Log-rank test.

Table 5 Correlation of micropapillary pattern, stromal invasion grade with overall survival by multivariate analysis in p-Stage IA cases

\begin{tabular}{lccc}
\hline Variable & Risk ratio & $95 \%$ CI & P-value \\
\hline Micropapillary pattern (positive) & 3.508 & $0.065-1.254$ & 0.0493 \\
Stromal invasion grade (grade 3) & 3.571 & $0.062-1.267$ & 0.0807
\end{tabular}

CI, confidence interval.

reproducibility of the classification in pT1 adenocarcinomas. Moreover, the concomitant use of micropapillary pattern improved the usefulness of the classification in predicting prognosis.

Micropapillary pattern is characterized by papillary structures with tufts lacking a central fibrovascular core. ${ }^{12}$ Patients with micropapillary patternpositive lung adenocarcinomas have poor prognosis and tend to present with extensive lymph node involvement and metastatic disease, ${ }^{12-15}$ similar to patients with micropapillary carcinomas of the breast ${ }^{23}$ urinary bladder, ${ }^{24,25}$ ureter ${ }^{26,27}$ and parotid gland. ${ }^{28,29}$ However, little is known about the mechanisms involved in micropapillary patternassociated lymph node metastasis. In our study, nodal metastasis, tumor size ( $>1 \mathrm{~cm}$ in diameter) and pleural invasion were significantly more frequent in micropapillary pattern-positive cases than in micropapillary pattern-negative cases. Furthermore, we demonstrated a close association between small cluster invasion observed in the portion of carcinoma invasion within fibrotic foci and presence of micropapillary pattern. We speculate that this may provide a new step towards elucidation of mechanisms involved in the lymphotropic feature of micropapillary pattern. Small cluster invasion is similar to tumor budding that was defined as small clusters of carcinoma cells lying ahead of the invasive front of the lesion in colon and rectal adenocarcinomas. ${ }^{18}$ It was shown that moderate or significant tumor budding correlated with more lymphatic and venous invasion and thus with a vigorous biologic activity of colon and rectal adenocarcinomas. ${ }^{18}$ Tumor dedifferentiation and dissociation at the invasive front may make it possible for carcinoma cells to invade blood vessels. Lung adenocarcinoma cells that show micropapillary pattern in alveolar spaces may tend to behave as small cluster invasion when involved in invasionrelated scar forming processes.

Only few studies have investigated the molecular changes associated with micropapillary pattern. Comparative genomic hybridization analysis in 16 micropapillary pattern-positive cases of invasive ductal carcinoma of the breast demonstrated uniformity of loss involving the short arm of chromosome 8 (8p). ${ }^{30}$ Candidate genes that have been proposed as driving the loss of $8 p$ region in solid tumors include EGR3, TRAIL receptors, DR4 and DR5, SCAM1, and the prostate-specific homeobox gene NKX3A. ${ }^{30}$ Relationship of loss of these genes with particular histologic features and the lymphotropic phenotype associated with micropapillary pattern is still unknown. Alteration of expression of cell surface molecules has also been shown to be associated with micropapillary pattern. For example, it is reported that MUC1 expression was predominantly demonstrated in the stroma-facing surface of the micropapillary cell clusters in micropapillary pattern-positive breast and colon carcinomas, and that this expression pattern indicates inversion of cell polarization as MUC1 is a glycoprotein normally located in the apical cell surface of normal glandular epithelium. ${ }^{31-33}$ It has been speculated that the presence of MUC1 in the stroma-facing surface of micropapillary cell clusters may lead to their inverted morphology and detachment of cells from the stroma based on the known function of MUC1 that inhibits the interaction between cell and stroma. $^{31-33}$

\section{References}

1 Kuroishi T, Nishikawa Y, Tominaga S, et al. Cancer mortality statistics in 33 countries (1954-1992). In: Tominaga S, Oshima A (eds). Gann Monograph on Cancer Res No. 47: Cancer Mortality and Morbidity Statistics. Japan Scientific Societies Press: Tokyo, 1999, pp 153-217.

2 Kakizoe T, Sugiura S, Segami K, et al. Cancer statistics in Japan 1999. Foundation of Promotion of Cancer Research: Tokyo, 1999, p 12.

3 Travis WD, Travis LB, Devesa SS. Lung cancer. Cancer 1995;75:191-202.

4 Kaneko M, Eguchi K, Ohmatsu H, et al. Peripheral lung cancer: screening and detection with low-dose spiral CT versus radiography. Radiology 1996;201:798-802.

5 Sone S, Takashima S, Li F, et al. Mass screening for lung cancer with mobile spiral computed tomography scanner. Lancet 1998;351:1242-1245.

6 Kurokawa T, Matsuno Y, Noguchi M, et al. Surgically curable 'early' adenocarcinoma in the periphery of the lung. Am J Surg Pathol 1994;18:431-438.

7 Noguchi M, Morikawa A, Kawasaki M, et al. Small adenocarcinoma of the lung. Histologic characteristics and prognosis. Cancer 1995;75:2844-2852.

8 Higashiyama M, Kodama K, Yokouchi $\mathrm{H}$, et al. Prognostic value of bronchiolo-alveolar carcinoma 
component of small lung adenocarcinoma. Ann Thorac Surg 1999;68:2069-2073.

9 Mountain CF. Revisions in the international system for staging lung cancer. Chest 1997;111:1710-1717.

10 Sobin L, Wittekind L. TNM Classification of Malignant Tumors. John Wiley \& Sons: New York, 1997.

11 Sakurai H, Maeshima A, Watanabe S, et al. Grade of stromal invasion in small adenocarcinoma of the lung: histopathological minimal invasion and prognosis. Am J Surg Pathol 2004;28:198-206.

12 Amin MB, Tamboli P, Merchant SH, et al. Micropapillary component in lung adenocarcinoma: a distinctive histologic feature with possible prognostic significance. Am J Surg Pathol 2002;26:358-364.

13 Miyoshi T, Satoh Y, Okumura S, et al. Early-stage lung adenocarcinomas with a micropapillary pattern, a distinct pathologic marker for a significantly poor prognosis. Am J Surg Pathol 2003;27:101-109.

14 Roh MS, Lee JI, Choi PJ, et al. Relationship between micropapillary component and micrometastasis in the regional lymph nodes of patients with stage I lung adenocarcinoma. Histopathology 2004;45:580-586.

15 Makimoto Y, Nabeshima K, Iwasaki H, et al. Micropapillary pattern: a distinct pathological marker to subclassify tumours with a significantly poor prognosis within small peripheral lung adenocarcinoma $(\leq=20 \mathrm{~mm})$ with mixed bronchioloalveolar and invasive subtypes (Noguchi's type C tumours). Histopathology 2005;46:677-684.

16 Travis WD, Brambilla E, Muller-Hermelink HK, et al. World Health Organization Classification of Tumors. Pathology and Genetics of Tumors of the Lung, Pleura, Thymus and Heart. International Agency for Reserch on Cancer Press: Lyon, 2004.

17 Eto T, Suzuki H, Honda A, et al. The changes of the stromal elastotic framework in the growth of peripheral lung adenocarcinomas. Cancer 1996;77: 646-656.

18 Hase K, Shatney C, Johnson D, et al. Prognostic value of tumor 'budding' in patients with colorectal cancer. Dis Colon Rectum 1993;36:627-635.

19 Shimosato Y, Suzuki A, Hashimoto T, et al. Prognostic implications of fibrotic focus (scar) in small peripheral lung cancers. Am J Surg Pathol 1980;4:365-373.

20 Yamashiro K, Yasuda S, Nagase A, et al. Prognostic significance of an interface pattern of central fibrosis and tumor cells in peripheral adenocarcinoma of the lung. Hum Pathol 1995;26:67-73.

21 Suzuki K, Yokose T, Yoshida J, et al. Prognostic significance of the size of central fibrosis in peripheral adenocarcinoma of the lung. Ann Thorac Surg 2000;69:893-897.

22 Maeshima AM, Niki T, Maeshima A, et al. Modified scar grade: a prognostic indicator in small peripheral lung adenocarcinoma. Cancer 2002;95:2546-2554.

23 Nassar H, Wallis T, Andea A, et al. Clinicopathologic analysis of invasive micropapillary differentiation in breast carcinoma. Mod Pathol 2001;14:836-841.

24 Amin MB, Ro JY, el-Sharkawy T, et al. Micropapillary variant of transitional cell carcinoma of the urinary bladder. Histologic pattern resembling ovarian papillary serous carcinoma. Am J Surg Pathol 1994;18:1224-1232.

25 Johansson SL, Borghede G, Holmang S. Micropapillary bladder carcinoma: a clinicopathological study of 20 cases. J Urol 1999;161:1798-1802.

26 Oh YL, Kim KR. Micropapillary variant of transitional cell carcinoma of the ureter. Pathol Int 2000;50:52-56.

27 Vang R, Abrams J. A micropapillary variant of transitional cell carcinoma arising in the ureter. Arch Pathol Lab Med 2000;124:1347-1348.

28 Michal M, Skalova A, Mukensnabl P. Micropapillary carcinoma of the parotid gland arising in mucinous cystadenoma. Virchows Arch 2000;437:465-468.

29 Nagao T, Gaffey TA, Visscher DW, et al. Invasive micropapillary salivary duct carcinoma: a distinct histologic variant with biologic significance. Am J Surg Pathol 2004;28:319-326.

30 Thor AD, Eng C, Devries S, et al. Invasive micropapillary carcinoma of the breast is associated with chromosome 8 abnormalities detected by comparative genomic hybridization. Hum Pathol 2002;33:628-631.

31 Pettinato G, Manivel CJ, Panico L, et al. Invasive micropapillary carcinoma of the breast: clinicopathologic study of 62 cases of a poorly recognized variant with highly aggressive behavior. Am J Clin Pathol 2004;121:857-866.

32 Nassar H, Pansare V, Zhang H, et al. Pathogenesis of invasive micropapillary carcinoma: role of MUC1 glycoprotein. Mod Pathol 2004;17:1045-1050.

33 Sakamoto K, Watanabe M, De La Cruz C, et al. Primary invasive micropapillary carcinoma of the colon. Histopathology 2005;47:479-484. 